\title{
Maximizing the Degree of Autarky of a 16 House Neighbourhood by Locally Produced Energy and Smart Control
}

\author{
Bart Homan ${ }^{\mathrm{a}, *}$, Gerwin Hoogsteen ${ }^{\mathrm{a}, *}$, Stefano Nebiolo ${ }^{\mathrm{a}}$, Johann L. Hurink ${ }^{\mathrm{a}}$, \\ Gerard J.M. Smit ${ }^{\mathrm{a}}$ \\ ${ }^{a}$ Computer Architecture for Embedded Systems, University of Twente, Enschede, the Netherlands
}

\begin{abstract}
In this work we investigate the possibilities of "soft-islanding" (near autarkic behaviour) a microgrid with 16 houses in the Netherlands, using the DEMKit simulation toolkit. We consider a situation where energy is generated by PV-panels as well as by a CHP and energy is stored using batteries as well as a heat buffer. This research is inspired by the Aardehuizen, which is a community in the Netherlands where the inhabitants strive to use as much as possible of their sustainably produced energy locally, motivated by the ideology that their community must act sustainably and self-sufficient, rather than by financial incentives. In a first step the proper size for the equipment is determined based on energy production and consumption data of several weeks in the year. Secondly one year simulations for several scenarios are presented and the degree of autarky for each scenario is compared. We demonstrate that a (nearly) autarkic operating microgrid can be achieved, with a proper but also with a realistic sizing of the components. Combining this proper sizing of energy generation and storage assets, with an advanced control, it is possible to achieve a degree of autarky of $99.1 \%$ over a year, meaning that less than one percent of the energy has to be imported from the main grid.
\end{abstract}

Keywords: (Smart)Microgrids, Energy management, Control, (Soft)Islanding, Storage

\section{Introduction}

With the current Energy Transition in the Netherlands, a country-wide operation is ongoing aiming at a structural change in energy usage to drastically reduce the emission of $\mathrm{CO}_{2}$ in the country [1]. One of the intended steps in the energy transition is to drastically reduce the usage of natural gas industrially, commercially and domestically. Focusing on domestic usage, most homes in the Netherlands are connected to the natural gas grid and this gas is used for space heating as well as domestic hot water (DHW) and for cooking [2]. As a result, currently most residences in the Netherlands are dependent

\footnotetext{
*Corresponding author, e-mail: \{b.homan, g.hoogsteen $\} @$ utwente.nl
} Preprint submitted to Sustainable Energy, Grids and Networks 
on natural gas. Simply switching to all electrical appliances is a difficult and costly process, for the end users as well as the network operators as the national electrical grid in the Netherlands is not designed for the resulting increase in electricity usage [3, 4].

To tackle the transition away from natural gas, there are two important other developments. On the one hand demanding loads, such as electric vehicles and heat pumps, may provide some flexibility to the system, e.g. by controlling the time for charging the electric vehicles or by using the thermal mass of the houses to control the heat-pumps. The objective of this control may e.g be to perform peak-shaving. Several control and optimization methods for energy management in the electricity systems are proposed in literature as surveyed by Siano [5]. In such a system, consumers receive incentives to which they can react in order to avoid overloading, or to increase the local consumption of renewable energy. However, the resulting effects depend heavily on the type of incentive used. McKenna and Keane [6] evaluated different incentive schemes and found that several uniform pricing schemes result in peak shifting rather than peak shaving if enough flexibility is provided. Furthermore, Nykamp et al. [7] state that steering by global market prices does not always reflect the local state of the grid and therefore amplify local negative effects. As a result, such schemes may even result in the need for more distribution and transmission capacity instead of a reduction.

On the other hand, locally produced energy may be used to reduce the burden for the electricity grid. For this an operational control mechanism is needed which aims to consume as much locally produced energy as possible [8]. In the scope of the electricity grid in this context the term microgrid was established, which was first coined by Lasseter [9]. A microgrid is a part of the electricity distribution grid that has the ability to temporarily disconnect from the national grid. This disconnected operation is called the islanded operation of a microgrid and improves the resilience of a grid by e.g. temporarily operating autarkic in case of failures in higher level parts of the grid hierarchy. Furthermore, in connected mode, a microgrid is able to provide ancillary services to the power grid such as acting as a virtual power plant [10]. Various control systems for microgrids have been presented in literature, see e.g. $[11,12]$. However, most control concepts mainly focus on optimizing the annual energy balance, which often leads to the implementation of a simple greedy strategy, which results in high import and export peaks to the main grid in smaller time scales. Another extreme is to create a fully islanded microgrid. But this is in general an appealing and costly solution. We propose a way in between by (nearly) islanding a microgrid from a power import and export perspective, but keep the grid connection for stabilization. This helps to avoid high investments e.g. associated to frequency balancing systems. Internally predictive and planning based control strategies are required to maximize the self-consumption of energy, and at the same time to minimize power peaks that are exchanged with the main grid, see e.g. [13, 14]. Such a connected microgrid is able to also act as a good citizen towards the national grid in order to defer associated grid investments.

Next to the electricity grid, also other energy carriers such as heat and gas should be integrated. Mancarella [15] has surveyed concepts and models for such integrated multienergy systems (MES), where the general conclusion is that a well organized MES is able to benefit from the synergies between the different energy sources and consumers. Economic optimization frameworks are proposed in [16] and [17], which show that a 
MES has indeed economic advantages. A trade-off between market optimization and local peak reduction using model predictive control in a MES is studied by Long et al. [18]. Their system uses a combined heat and power (CHP) unit, a heat pump and a storage to provide flexibility. They conclude that also for MES other incentive schemes are required to make a positive case for local peak reduction, which agrees with the conclusion by [7]. Note that the choice of a proper MES solution may be very climate specific. For example; Perez et al. [19], optimize a Texas based MES, where cooling is dominant instead of heating, although the setting is quite different they draw similar conclusions concerning the energy market.

Inspiration for this work comes from the Aardehuizen community [20] in Olst, the Netherlands. The members of this community strive towards living responsibly with respect to the environment. In compliance with this, their houses are built using sustainable materials and their goal is to fulfill the energy needs by sustainable sources (e.g. by using PV-panels) where possible. Not only on a yearly basis, but by balancing energy locally at all times. However, as this is not reached completely, a connection to the national electricity grid is still needed to balance the local grid. For example to fulfill the demand when the local production from renewables is not sufficient, or to feed-in overproduction by PV panels. Note that, as indicated in Figure 1 the share of energy currently used in the Netherlands originating from renewable sources is small $(\sim 5 \%)$. Hence the operation of a migrogrid would be idealy suitable for the Aardehuizen community to limit imports of non-sustainably generated energy from the grid. Note that in this case the move towards a microgrid is driven by the ideological beliefs of the Aardehuizen members, rather than by financial incentives.

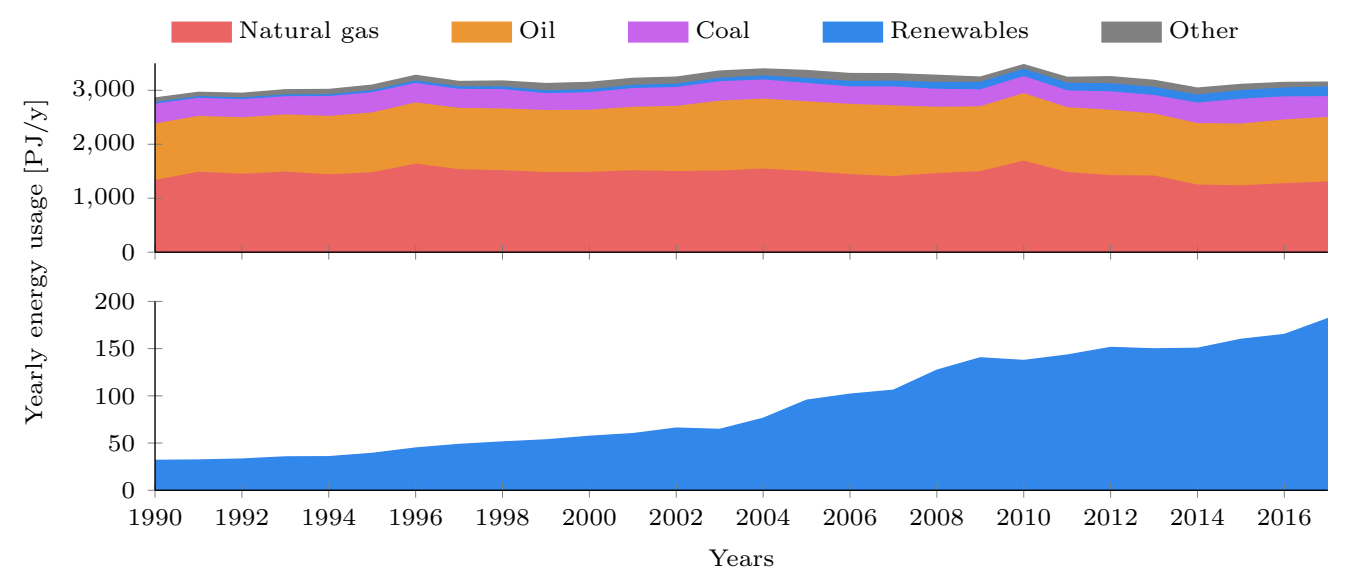

Figure 1: Yearly energy usage in the Netherlands, divided by energy carrier. Data obtained from the Dutch Central Bureau of Statistics [21]

In previous work [22] we analysed a MES and coined the term "soft-islanding" for such a a nearly islanded microgrid. The results of that study show that there is potential in creating a reasonable soft-islanded microgrid, consisting of 16 households, that can operate nearly autarkic using locally produced renewable energy from photovoltaic (PV) panels and a centrally located CHP. Thereby, only $2 \mathrm{kWh}$ battery storage capacity was 
required per household. However, the setup of the system and the used models in that study are quite simple and it is not completely clear if the achieved results are realistic and are valid under all conditions.

Therefore we revisit the original case study presented in [22] and use refined models to obtain more realistic results under challenging conditions. These refined models include the addition of first order thermal models, an advanced and validated battery model, and a synthetic load-profile that is validated using measurement data collected in a fieldtest. Also the optimization algorithms are improved to use real predictions, whereas the control mechanism now also deals with prediction errors that arise. The goal of our research is to perform a technology driven study to investigate the technical feasibility of such a MES, together with the control system. A method to size the required local generation and storage units to make soft-islanding possible under realistic circumstances is therefore presented in this paper. For this we use a futuristic "greenfields" scenario, with full flexibility to arrange the layout of the neighbourhood and use best-practices in construction of houses, such as e.g. using high insulation standards.

With the technology driven approach, we intend to make this study independent from current (local) market models, (local) tax schemes and market prices of technology. The technical blueprint for an (nearly) autarkic micro-grid presented in this paper therefore has no bias to a particular energy market or regulatory framework. Instead, the results can be used as input for an economic (feasibility) study within a given context of (local) energy markets, prices, and other local aspects. Additionally, the technical analysis can be used as input for discussions on how to shape, amongst others, future market models, $\left(\mathrm{CO}_{2}\right)$ tax schemes, and regulations.

The remainder of this paper is organized as follows. Section 2 introduces terminology and presents the research method, including the used software, parameters and models. A case description of the neighbourhood is given in Section 3. The sizing of all components, using a simultaneous sizing approach is described in Section 3.4. In Section 4 the results of simulation studies are presented. Conclusions are discussed in Section 5, followed by topics for future work in Section 6.

\section{Tools \& Methods}

The goal of this paper is to research the possibility of generation and storage devices within a soft-islanded microgrid. In order to evaluate whether or not a microgrid can be considered as being soft-islanded, a clear definition of soft-islanding is required. As stated in the introduction, a soft-islanded grid should be able to operate nearly autarkic, i.e. the exchange of energy with the main grid should be minimized and should ideally be zero. Furthermore, customers connected to the microgrid should not experience drawbacks of being connected to such a soft-islanded microgrid compared to a conventional grid. Hence, e.g. load shedding is not an option, as comfort of end-users must be preserved. However, we note that e.g. production curtailment of PV panels may be applied to avoid large exports to the main grid.

To achieve the stated goal, we have to focus on the energy that is locally produced and therefore does not need to be imported from the main grid. A frequently used term for 
this is the self-consumption, which is defined as the ratio between the energy consumed directly in the microgrid and the total produced energy. The drawback of this metric is that it can result in a relative low value when an overcapacity of energy producing devices, such as PV, is installed. However this is not very grid friendly, as this might lead to a high export of electricity. On the other hand, to reduce the import from the main grid, installing an overcapacity of PV panels may be an economic appealing solution and may be preferable to installing more battery capacity for this goal. Another metric that could be used to compare different solutions is the Demand Cover Factor ${ }^{1}$ (DCF) as formulated by e.g. Palacios-Garcia et al. [25]. However the DCF as introduced is not completely suitable for our situation, since it only uses PV as a source of local energy generation. As the focus of this paper is on MES, with multiple sources, we introduce and use a generalized metric that is technology independent, the Degree of Autarky (DoA). The DoA is defined as the percentage of energy consumption ( $\left.\mathrm{E}_{\text {consumption }}\right)$ from local sources, i.e.

$$
\text { DoA }=\frac{\mathrm{E}_{\text {consumption }}-\mathrm{E}_{\text {import }}}{\mathrm{E}_{\text {consumption }}} \times 100 \%
$$

where $\mathrm{E}_{\mathrm{import}}$ is the total amount of imported energy. In contrary to the self-consumption, the DoA value does not decrease when overproduction (the part that is being exported) is installed. Note, that by matching supply and demand within the microgrid, e.g. by using a storage device, the DoA can be increased.

For us to consider a microgrid to be soft-islanded, we aim for an as high as realistically possible DoA. However, note that the last few percent points of DoA usually lead to very high investments, such as large batteries, of which the full capacity is only used in rarely occurring situations. Thus for our greenfields case we aim for a DoA of at least $98 \%$, which means that the neighbourhood needs to import electricity from the grid for at most a total of one week in the year. Furthermore, with the ongoing electrification in mind, we focus only on the interaction with the electricity grid. It is assumed e.g. that abundant (bio) fuel is available in the vicinity to power a CHP.

\subsection{Method}

For this research we follow to a large extend the same basic method and setup of 16 houses as in [22]. The results of that study indicate that $15 \mathrm{~m}^{2} \mathrm{PV}$-panels and a $2 \mathrm{kWh}$ battery per house, in addition to a shared $60 \mathrm{kWh}_{\text {th }}$ (thermal) / $30 \mathrm{kWh}_{\mathrm{e}}$ (electric) CHP with a $250 \mathrm{kWh}$ heat buffer, is enough to achieve semi-autonomous operation from the grid. The simulation studies for that paper were executed using the TRIANA simulation software $[26,27]$, whereas optimization was performed using the profile steering approach presented in [14]. However, the results of this case-study are obtained with ideal, loss-free, models and the optimization methodology used perfect predictions of energy consumption. In this way the achieved results are based on not very realistic models and assumptions.

To achieve a more realistic approach to size the soft-islanded neighbourhoods, we first define a scenario with fixed parameters for the considered devices. For this scenario a

\footnotetext{
${ }^{1}$ In literature this metric is also referred to as Load Cover Factor [23] and Load Match Index [24].
} 
parameter sweep is executed using the DEMKit simulation and demonstration toolkit [28]. The parameters for which this sweep is executed are the PV panel area, the battery capacity and the heat to electricity ratio of the CHP (discussed in Section 3). Based on the appropriate sizes for each of these components, different control strategies ([14] and [29]) are used to evaluate the setting. The remainder of this section presents the tools and refined models used to simulate the soft-islanding neighbourhood.

\subsection{Artificial Load Profile Generator}

A crucial part of the simulations is the input data. Large parts of the required data is associated with the electricity and heat demand of the 16 houses. This kind of data generally is not readily available, and even when it is, it is very privacy sensitive and its usage is restricted by privacy laws and regulations. Therefore statistics or models are used to generate artificial profiles for the electricity and heat demand of households. A comprehensive review by Torriti [30] outlines that many different methods and approaches to obtain or generate such profiles are described in literature. For example, a detailed bottom-up stochastic model for electricity usage is proposed by Wilke et al. [31]. López-Rodríguez et al. [32] use a time-of-use survey to construct electricity consumption profiles for households, while a more general method to construct profiles for electricity and hot water usage is demonstrated by Wíden et al. [33, 34]. Note, that the mentioned methods and approaches form only a brief selection of the many possible approaches presented in literature.

Our input data, i.e. the profiles for the electricity and heat demand, are generated using the Artificial Load Profile Generator (ALPG) presented in [35]. This open source profile generator uses a bottom-up modelling approach where the behaviour of individual inhabitants is simulated to obtain an occupancy profile. Resulting output of this generator was previously validated to accurately resemble real measurement data that was collected in a neighbourhood for a timespan of a year. The output of this ALPG results in a time series load profile for uncontrollable loads and vectors of constraints for devices that offer flexibility, such as e.g. start times (the moment the device is available) and end times (the deadline for the device to finish) for a washing machine. The control system uses these constraints on the usage of these devices to optimize their actual run times. A possible goal for this optimization may be to match electricity demand with the production by PV and the CHP.

The original ALPG only supported electricity profiles. Therefore we have extended its functionality with heat demand generation models. Now it also produces a vector with thermostat set-points to express the desired indoor temperature. Furthermore, the ventilation profile is linked to the occupancy profile to ensure that enough fresh air is brought into the house. Similar to the electricity consumption profile, also domestic hot tap water usage profiles are generated. This information, together with weather data, is used as input for the thermal model of a household as implemented in DEMKit. The household types used to generate the load profiles for the model of the greenfields neighbourhood are listed in Table 1. Furthermore, for the thermal simulation and calculation of PV production, weather data from the KNMI [36] for 2013-2014 was used from weather station Twenthe. 
Table 1: Households family type composition of the 16 houses

\begin{tabular}{c|l}
\hline \# of houses & Family Type \\
\hline 6 & HouseholdDualWorker \\
1 & HouseholdFamilySingleWorker \\
5 & HouseholdFamilyDualWorker \\
1 & HouseholdSingleRetired \\
2 & HouseholdDualRetired \\
1 & HouseholdDualWorker with one worker only \\
\hline
\end{tabular}

\subsection{DEMKit}

To perform the case-studies as presented in this paper, we use the DEMKit (short for Decentralized Energy Management ToolKit) simulation and demonstration software developed at the University of Twente [28]. The DEMKit provides a library of device components (amongst others, electric vehicles and static loads), physical models (e.g. grid infrastructure) and control systems with optimization algorithms. Herein, DEMKit uses a bottom-up cyber-physical systems approach where these components influence each other through their relations. As such, all components together form a (micro) grid whose behaviour can be simulated in detailed discrete time steps. DEMKit is the successor of the TRIANA simulator [26, 27] used previously, but is completely rewritten in Python. Both stability and simulation speed have been improved, but also new control functionality is introduced.

New device components have been added to improve the accuracy of the simulation results. A realistic battery model based on models presented in [37], which can be used as replacement for the ideal battery model used in [22]. The battery optimization and control remains the same due to the standardized model interface [38]. Thermal models, which are extensively evaluated in [39]. Two models are used here, a 1R1C model (one resistor, one capacitor) for conventional heating systems with radiators and a $2 \mathrm{R} 2 \mathrm{C}$ model used for floor heating systems as found in modern and well insulated households. Coupled to such a zone are a heat source, such as a CHP or heat network, and a thermostat.

Control and optimization techniques have also received a major update in DEMKit compared to the approach used in the original paper [22]. Firstly is the addition of prediction modules for consumption, production and the expected flexibility that a device can offer based on historical data. These predictions are used as input for the optimization process, which is the profile steering algorithm [14]. The profile steering approach is extended with an event-driven approach which allows to react on a deviation from the planning directly at the moment such a deviation occurs [40]. This way, the realistic performance of the control system approach can be tested. Furthermore, an online control system based on double-sided auctions [29] is introduced, which uses the same control structure of control as used with profile steering.

\subsection{Coordination mechanisms}

To achieve a soft-islanded microgrid, it is of utmost importance to coordinate the energy production, storage, and consumption between the various assets available within the microgrid. Within this paper we use the profile steering algorithm to optimize the energy 


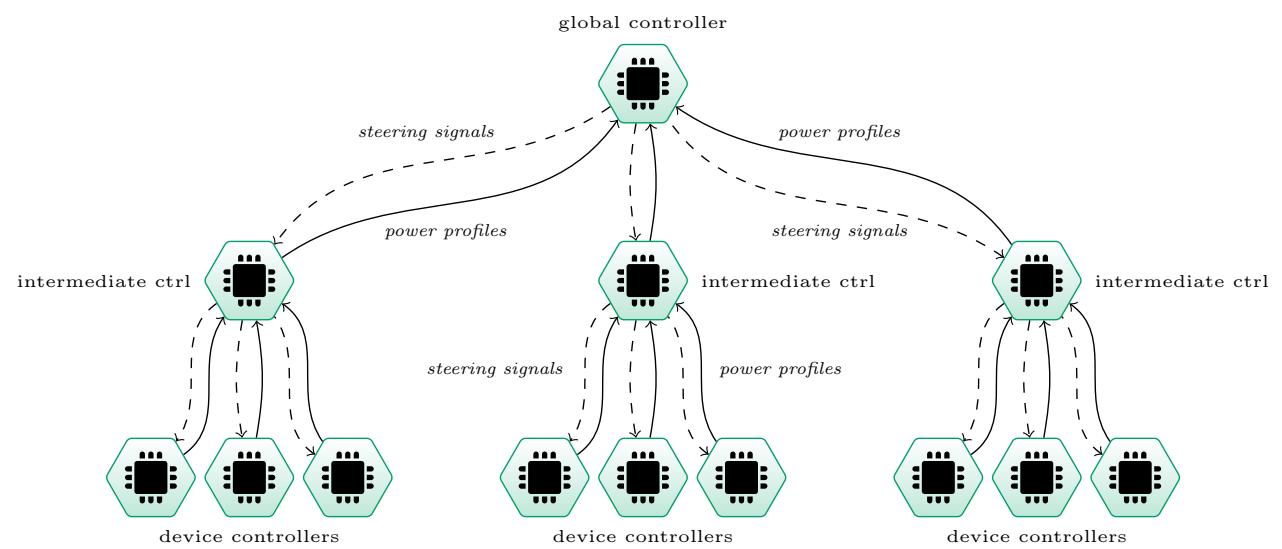

Figure 2: Hierarchical structure of higher level controllers running profile steering and device controllers as leaves.

profile of the microgrid. The coordination structure consists of a hierarchical tree, in which the leaves are controllers representing a specific, individually modeled, device. All other (intermediate) nodes are formed by controllers running the profile steering algorithm to coordinate the energy profile of their respective children (which may consist of nodes running profile steering too). This structure is depicted in Figure 2.

Profile steering is an optimization framework that works by means of a goal profile $\vec{p}$ which specifies the desired overall power consumption/production of the whole neighbourhood for $N$ discrete time intervals in the future, i.e. $\vec{p}=\left[p_{1}, p_{2}, \ldots, p_{N}\right]^{T}$. In an iterative manner, the profile steering controllers coordinates the overall power profile $\vec{x}=\left[x_{1}, x_{2}, \ldots, x_{N}\right]^{T}$ of the neighbourhood. This power profile is the aggregated profile of all individual children in a set $M$, i.e. $\vec{x}=\sum_{m \in M} \overrightarrow{x_{m}}$. In each iteration, each child proposes a new power profile $\overrightarrow{x_{m}}=\left[x_{m, 1}, x_{m, 2}, \ldots, x_{m, N}\right]^{T}$, which expresses a potential power consumption/production for child $m$ in each interval to improve the objective at the parent. Within this paper, we use as objective to minimize $\|\vec{x}-\vec{p}\|_{2}$. The profile steering node iteratively selects the child that improves the objective most, resulting in updated profiles $\vec{x}$ and $\overrightarrow{x_{m}}$. This iterative process continues until no (significant) improvements are possible. We refer the reader to [14] for more details on the profile steering algorithm. Device specific optimization algorithms suitable with profile steering are presented by Van der Klauw [41].

The presented optimization framework relies on predictions (e.g. of solar production or local constraints) as it optimizes the energy profile for future intervals. These predictions are never accurate, possibly resulting in infeasible schedules for some devices. However, executing a new planning upon each and every deviation is computationally too expensive. Therefore, two online control methods to resolve prediction errors are considered in this context.

The first is event-based profile steering [40], to which we refer as PSE in this work. This method utilizes the overall planning $\vec{x}$ created by the normal profile steering algorithm 
as a guideline. Additional vectors are introduced, which contain the most actual profiles of the connected children, denoted by $\vec{m}$, and the aggregated profile a the parent profile steering node $\vec{r}=\sum_{m \in M} \overrightarrow{r_{m}}$. Upon major events in the local (device) setting, a child $m$ sends a signal to its parent. These major events include e.g. weather forecast updates, a washing machine that becomes available, or upon detection that the existing planning becomes infeasible. The parent node responds to $m$ with the predicted deviation of its current planning $\vec{x}$, i.e. $\vec{r}-\vec{x}$ is communicated back to $m$. Now, the objective for the device is to minimize $\|\vec{r}-\vec{x}\|_{2}$ by creating or updating planning $\overrightarrow{r_{m}}$, whereby $m$ replaces its old planning $\vec{m} \vec{m}$. In this way, the neighbourhood profile stays as close to the original planning as possible. Since only one device needs to be (re-)planned at a time, no iterations are required and this approach is able to repair infeasible solutions using the same code-base.

The second method is to combine profile steering with a double-sided auction [29], to which we refer as PSA in this work. The overall planning $\vec{x}$ as a result of the profile steering algorithm serves as a guideline in this method. The task of the double-sided auction is to realize this planned power level $\overrightarrow{x_{n}}$ by performing an auction every time interval $n$. For each auction, all devices construct a bid and communicate this bid to the auctioneer. A bid is a function that specifies the amount of power a device will consume/produce in a give time interval for each price in a given set of prices. Furthermore, these bids only contain feasible power options for each device and take into account local preferences. The auctioneer aggregates all received bids and subsequently selects a price for which the aggregated power consumption is closest to $\overrightarrow{x_{n}}$. This price is communicated to the devices, which in turn update their power consumption as specified by their bids. In contrast to the aforementioned event-based approach, the double-sided auction is able to control all devices in one auction round, but loses the ability to consider effects on the energy usage in future intervals.

The desired profile used as objective in this work is the so-called zero-profile (i.e. $\vec{p}=$ $[0, \ldots, 0]^{T}$ ), which indicates that energy should be kept locally as much as possible and that the profile of imported or exported energy $(\vec{x})$ should be flattened. Each planning spans 192 intervals of 15 minutes (i.e. 2 days) and a rolling horizon approach is used, in which a new planning is created every 96 intervals (i.e. every day). The simulations conducted in this work cover a complete year (365 days), where the discrete simulation time step (interval length) is set to 900 seconds (15 minutes). We refer the reader to [28] more details on the planning and prediction algorithms.

\section{The layout of the neighbourhood}

The neighbourhood considered for this research (see Figure 3) is in many respects ideal. It consists of 16 houses arranged around a square. All houses are newly built and well insulated. The residents are assumed to be fully co-operative, they are willing to share data regarding their energy usage and behaviour and willing to share electricity, heat and flexibility.

\subsection{Neighbourhood characteristics}

All houses have a large section of windows at the back side of the house, facing the square and a smaller section of windows at the front side of the house, facing outward. So, based 


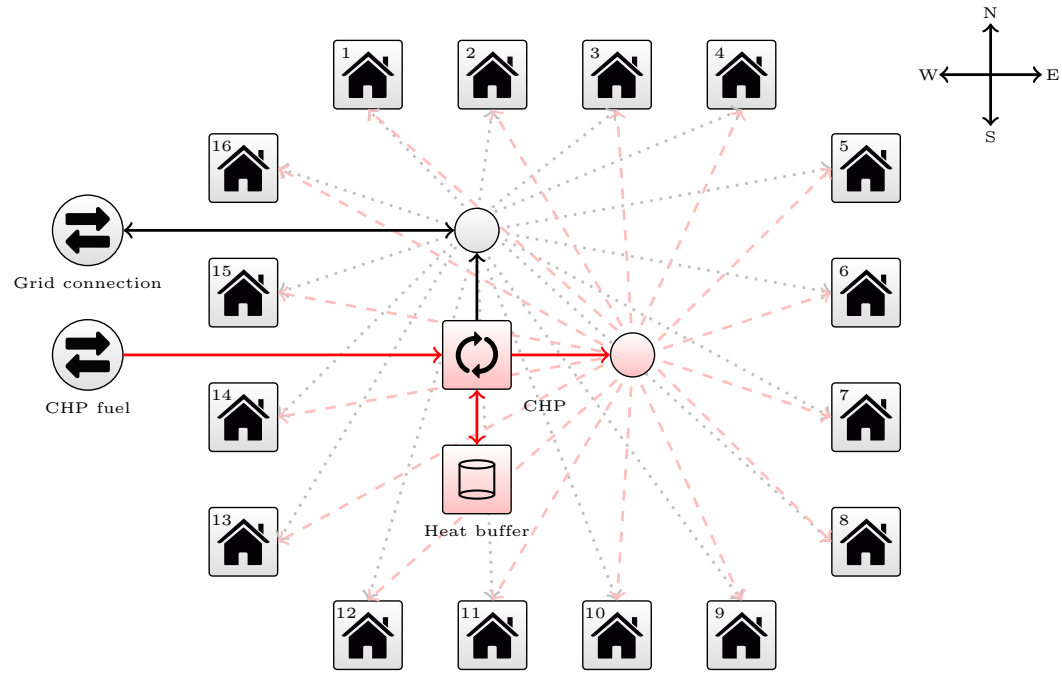

Figure 3: Schematic representation of the arrangement of the individual houses in the neighbourhood. The connections necessary for the distribution of heat (red) and electricity (black) are also displayed.

on this arrangement there are four types of houses to consider, those facing North, East, South and West. For instance, houses 1,2,3 and 4 are of type North. These houses have a large section of windows $\left(12 \mathrm{~m}^{2}\right)$ facing South, a smaller section of windows $\left(8 \mathrm{~m}^{2}\right)$ facing North and all the PV-panels are on the south side of the roof. The other house types have similar but slightly different characteristics, which are outlined in Table 2.

Table 2: Characteristics of the different house types in the neighbourhood.

\begin{tabular}{l|llll}
\hline Type of house & North & East & South & West \\
\hline House \# & $1,2,3,4$ & $5,6,7,8$ & $9,10,11,12$ & $13,14,15,16$ \\
Front windows & $8 \mathrm{~m}^{2} \mathrm{~N}$ & $8 \mathrm{~m}^{2} \mathrm{E}$ & $8 \mathrm{~m}^{2} \mathrm{~S}$ & $8 \mathrm{~m}^{2} \mathrm{~W}$ \\
Back windows & $12 \mathrm{~m}^{2} \mathrm{~S}$ & $12 \mathrm{~m}^{2} \mathrm{~W}$ & $12 \mathrm{~m}^{2} \mathrm{~N}$ & $12 \mathrm{~m}^{2} \mathrm{E}$ \\
PV location & $\mathrm{S}$ & $\mathrm{E}$ & $\mathrm{S}$ & $\mathrm{W}$ \\
PV angle & $35^{\circ}$ & $35^{\circ}$ & $35^{\circ}$ & $35^{\circ}$ \\
PV efficiency & $16 \%$ & $16 \%$ & $16 \%$ & $16 \%$ \\
\hline
\end{tabular}

There are also some facilities shared by the whole neighbourhood. Firstly, there is one connection to the grid outside the neighbourhood, which is used to import or export electricity if required. Secondly, the neighbourhood shares a Combined Heat and Power generator (CHP). The CHP can be fuelled using various types of fuel, e.g. wood pellets, (bio)-gas or (bio)-oil, however, the type of fuel is not specified in this study. The CHP generates electricity which can be distributed to all houses. The CHP also generates heat in the form of hot water which is also distributed to all houses by a local heat network. The CHP has a $250 \mathrm{kWh}$ heat buffer, which effectively decouples the heat and electricity production by the CHP. If electricity is desired when there is no heat demand, the produced hot water is stored in the buffer. Conversely, if hot water is 
desired when there is no electricity demand, hot water can be discharged from the buffer to meet the demand. The main goal for the CHP is to generate enough heat to meet the heat demanded by the 16 houses at all times. The electricity generated by the CHP is only a by-product which is used to meet part of the electricity demand. The rest of the electricity demand is met by electricity produced by the PV-panels and by using the battery optimally. In case of an electricity shortage, input from the grid is used as a last resort.

Figure 4 gives a schematic representation of the composition of the individual houses. The square icons represent the devices and energy demands. Each house has a heat demand consisting of both space heating and tap water and it also has fixed and flexible loads. Fixed loads are devices that are switched on at a time that is convenient for the user, e.g a lamp or coffee maker. Flexible loads are devices that can be switched on or off at specific times, as long as their function fulfils the requirements of the users (e.g. a washing machine or a dishwasher should be finished at the time specified by the user). Furthermore, each house contains a battery for storage of electrical energy, and a set of $\mathrm{PV}$-panels to generate electrical energy.

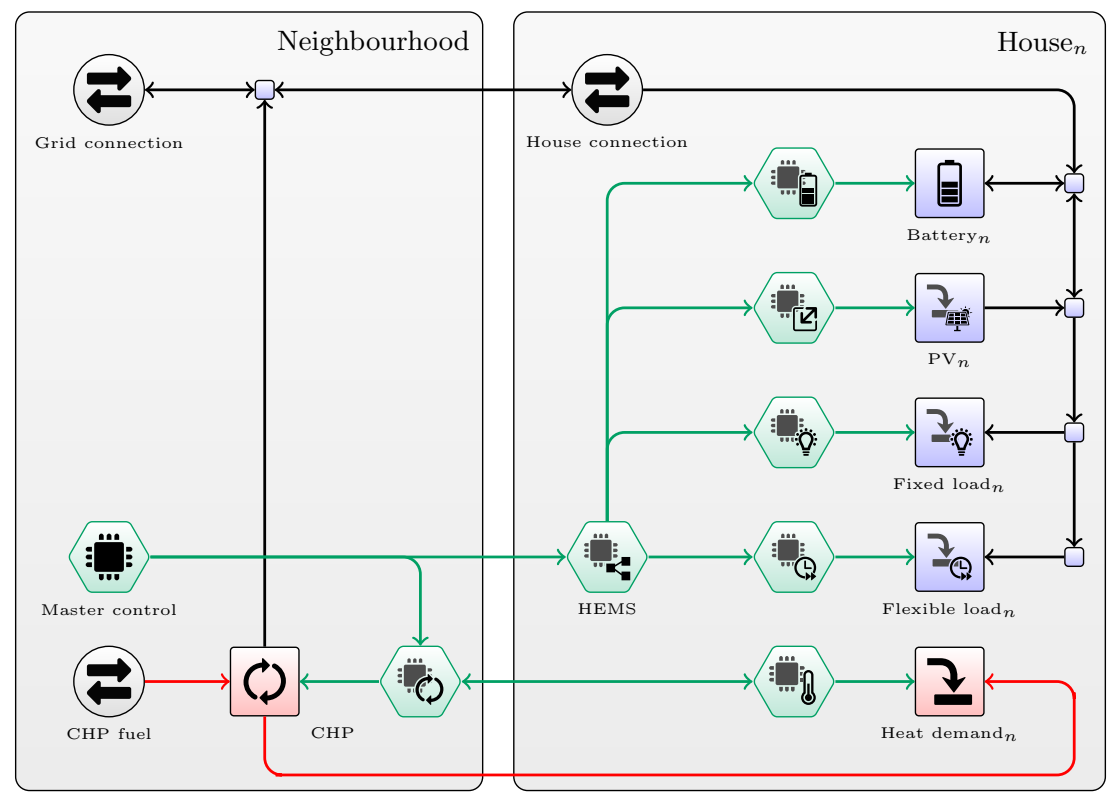

Figure 4: Schematic representation of an individual house and its connection to the central CHP and control system. The physical part of the model is represented by square icons (devices) and circular icons (grid infrastrucure). The cyber part is shown using hexagonal icons that represent (embedded) computers that run the control algorithms. The links between these icons depict the relation between two or more objects.

The hexagonal icons in Figure 4 represent (embedded) computers that run the control algorithms. For instance a battery controller is used to control the battery, meaning that the state of charge ( $\mathrm{SoC})$ is monitored and that commands are given on how much energy should be stored in the battery or be discharged from the battery. A time-shiftable controller is used to control flexible devices, for which it is possible to control the start of 
the operation of such a device within given limits. All controllers in a house are in turn controlled by the home energy management system (HEMS). The HEMS also accepts (overriding) input from the residents. Lastly, the master controller controls the HEMS's in the neighbourhood as well as the central CHP to make sure that all energy demands are met in such a way that a minimal amount of energy is imported from the grid.

\subsection{Proper size of relevant equipment}

As shown in Figure 4, each house in the Greenfields neighbourhood has PV-panels and a battery, and shares a CHP with the other houses, but the proper size of these components has yet to be determined. In the previous research [22] it was concluded that using 15 $\mathrm{m}^{2} \mathrm{PV}$-panels and a $2 \mathrm{kWh}$ battery per house, and a $60 \mathrm{~kW}$ thermal / $30 \mathrm{~kW}$ electric CHP with a $250 \mathrm{kWh}$ buffer, might be enough to achieve soft-islanding. However, a preliminary simulation of the Greenfields neighbourhood with the extended models, using these parameters did not support this result. There are several days in the year, during which there is still a significant power demand needed from the grid. Therefore, in a first step new specifications for the equipment have to be determined with which the neighbourhood can achieve soft-islanding.

We use simulations to determine the size of equipment in the given neighbourhood. More specifically, we use a complete model of the neighbourhood, consisting of individually modeled devices and controllers as presented in the previous subsection. Several simulations are executed, in which certain parameters are changed, such as the capacity of batteries or the size of a PV setup. Furthermore, we use the PSE energy optimization method (presented in Subsection 2.4), including predictions. The resulting DoA of the simulations are used to select a proper size of the equipment. The advantage of this method is that the same control is used during the sizing step, such that it accurately represents the final energy profile, and thus DoA, of the considered soft-islanded community. The drawback is that this method is time consuming due to the vast amount of detailed simulations that need to be executed. Note that simply scaling the achieved results is not an option as different sizes of equipment often result in a different use of flexibility. This is the result of the complex interaction with other equipment or to deal with e.g. uncertainty.

\subsection{Reconsidering the battery size}

In [22], the size of the home batteries was the decision which was made based on already given specifications of the other devices, thereby it's capacity was chosen as the smallest capacity necessary for achieving soft-islanding. Therefore a logical first step is to analyse whether or not simply increasing the battery size is enough to achieve soft-islanding with the new setting.

To determine the appropriate battery size three weeks were analysed in detail, week $A$; a week during which the highest PV-production in the year is achieved (in May), week B; a week during which there is low PV-production as well as a low heat demand (in October) and week $C$ a week during which the highest heat demand of the year is achieved (in November). The characteristics of these weeks are listed in Table 3.

In week $A$ there is a high generation of electricity by the PV-panels, and a low heat demand. In Figure 5a the grid power demand for the whole neighbourhood in week A is 
Table 3: Description of the test weeks with the energy production in these weeks.

\begin{tabular}{l|lllll}
\hline Week & Date & \multicolumn{2}{l}{ PV generation } & \multicolumn{2}{l}{ Heat demand } \\
\hline A & $7-15$ May. & highest & $(194 \mathrm{kWh} /$ panel $)$ & low & $(678 \mathrm{MWh})$ \\
B & $2-9$ Oct. & low & $(70 \mathrm{kWh} /$ panel $)$ & low & $(706 \mathrm{MWh})$ \\
C & 27 Nov. -3 Dec. & low & $(20 \mathrm{kWh} /$ panel $)$ & highest & $(7853 \mathrm{MWh})$ \\
\hline
\end{tabular}

shown (negative demand being exported) for different sizes of the house battery between $2 \mathrm{kWh}$ and $6 \mathrm{kWh}$. Figure 5b shows the corresponding combined SoC of the batteries. In case of the smallest battery capacity $(2 \mathrm{kWh})$, the electricity generated by the CHP and the PV-panels is more than sufficient to meet the daytime demands as well as charging the battery completely (see Figure 5b). In the evening when there is no generation from the PV-panels, however, the electricity generated from the CHP and discharged from the batteries is sometimes insufficient to meet the demands. If the battery capacity is large enough also the electricity demands in the evening can be met easily. From the figure it is clear that a battery size of $5 \mathrm{kWh}$ is sufficient.

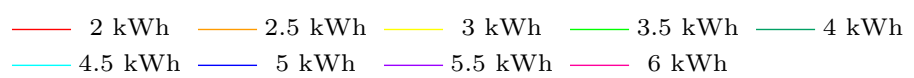

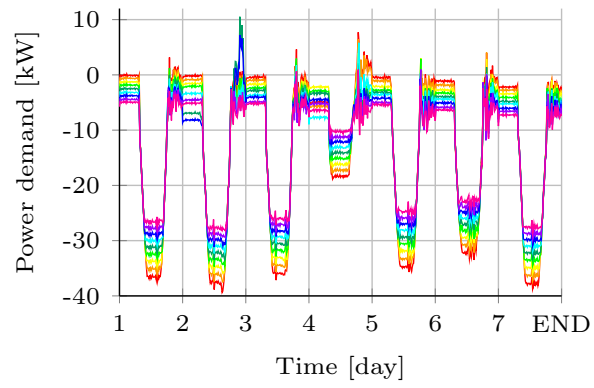

(a) Week A: Total grid power demand

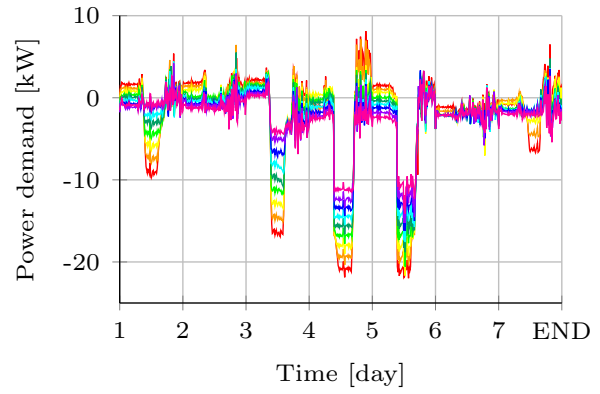

(c) Week B: Total grid power demand

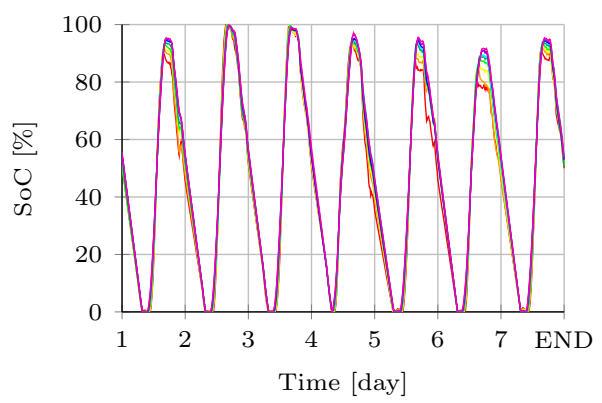

(b) Week A: Combined SoC

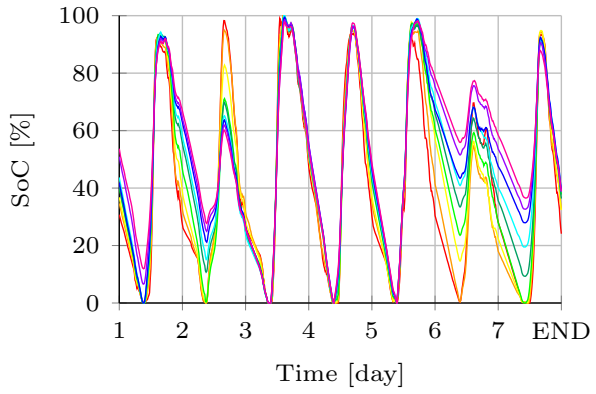

(d) Week B: Combined SoC

Figure 5: Simulation of the total grid power demand and combined battery state of charge for several battery capacity in the test weeks A and B. Note that the grid power demand is displayed, so a negative power demand is power supplied back to the grid. 
In week $B$ there is little generation of electricity by the PV-panels and a low heat demand. Figure 5c shows that the electricity generated by the CHP and the PV-panels combined with a $2 \mathrm{kWh}$ battery is only sufficient to meet the electricity demands during the day. In this case even increasing the battery capacity to $6 \mathrm{kWh}$, which is 3 times the original capacity, does not solve all the problems. On the days where the least electricity is generated by the PV-panels (most notably day 6 ) the battery can not be fully utilized (see Figure 5d). However, on the days where the most electricity is generated by the PV-panels on average $15 \mathrm{~kW}$ is delivered back to the grid. To get more insight in the usage of the grid Figure 6 gives the amount of electricity imported from the grid and for the different battery capacities and the two weeks A and B, we see that the import decreases as the battery size is increased, however, with each increase in battery size there is a smaller decrease in electricity imported from the grid. Hence, at some point the decrease of electricity imported from the grid does no longer justify the costs of the necessary battery capacity. So in this case increasing the battery capacity will not yield any improvements, unless an unjustifiably large battery is used.

In week $C$, the electricity generated by the $\mathrm{CHP}$ is more than sufficient to meet the electricity demands regardless of the amount of electricity produced by the PV-panels or the capacity of the battery.

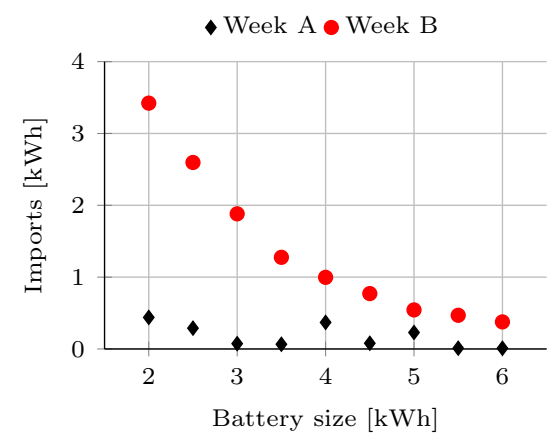

Figure 6: Total amount of electricity imported from the grid in test weeks A and B for different battery sizes.

\subsection{Simultaneous sizing of the battery, pv-panels and CHP.}

To decrease the import from the grid to almost $0 \mathrm{~kW}$, increasing the battery capacity would only be effective in week $A$. In week $B$ even an increase of the battery capacity by a factor of 3 would have only a limited positive effect and still a positive grid power demand remains. In week $C$ the grid power demand is already zero regardless of the battery size. However, the battery is not the only device which can be optimized to decrease the grid power demand to zero. The area of PV-panels per house, and the heat / electricity production ratio of the CHP can also be optimized. Therefore for these two parameters a parameter-sweep was performed. In Figure 7 the average daily grid energy demand per house in week B, as a function of the PV-panel area for five battery capacities and three different CHP heat / electricity production ratios is displayed.

The trend in all three figures is that the maximum grid power demand is decreasing with 


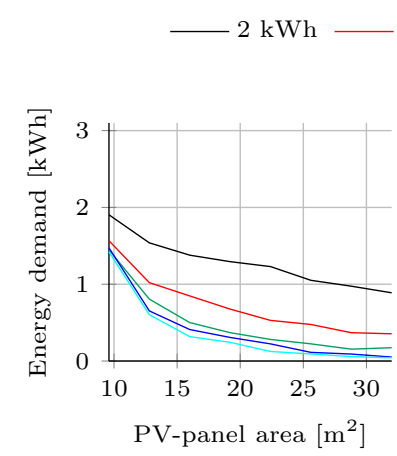

(a) $\mathrm{CHP} h / \mathrm{e}=1.75$

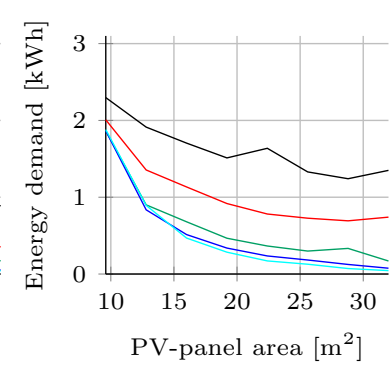

(b) $\mathrm{CHP} h / \mathrm{e}=2.0$

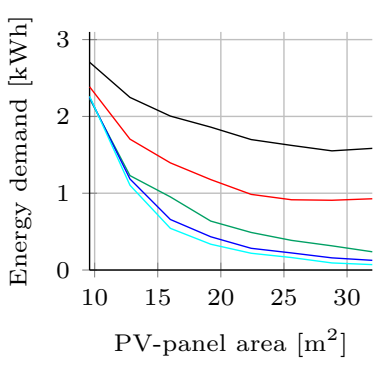

(c) $\mathrm{CHP}$ h/e $=2.25$

Figure 7: Average daily grid energy demand per house for the test week A as a function of PV-panel area for several battery sizes and CHP heat / electricity production ratios.

an increase in PV-panel area. In all three figures the maximum grid power demand is also smallest for the largest battery size. Increasing the battery capacity from $2 \mathrm{kWh}$ to $3 \mathrm{kWh}$ or from $3 \mathrm{kWh}$ to $4 \mathrm{kWh}$ results on average in a $0.5 \mathrm{kWh}$ decrease of the average daily grid energy demand per house. A further increase of the battery capacity results only in a limited decrease. Lastly, the average daily grid energy demand per house is smallest for the smallest CHP heat / electricity production ratio 1.75 (Figure 7a), however, the CHP heat / electricity production ratio has a very limited influence on the average energy demand per house.

The average daily grid energy demand per house in test week $A$ is always below 0.5 $\mathrm{kWh}$. Moreover increasing the PV-panel area or the battery capacity, or changing the CHP heat / electricity production ratio yields only small improvements. The average daily grid energy demand per house for test week $\mathrm{C}$ is always below $0 \mathrm{kWh}$, regardless of the CHP heat / electricity production ratio, PV-panel area or battery capacity. So the parameter sweep analysis of weeks $A$ and $C$ had no influence on the equipment sizing choices.

In conclusion, the power demanded from the grid can be decreased by increasing the battery capacity, however, the further the battery capacity is increased, the lower is the decrease of the power demand from the grid (see Figure 6). Hence, if only the battery capacity is considered to decrease the grid power demand in week B (low PV production and low heat demand), even a capacity of $6 \mathrm{kWh}$ per house is not enough to decrease the power demand from the grid to zero. Moreover the increased battery capacity is only needed for a few days each year, while the rest of the year it would remain unused. So this would not be a very efficient solution. A better solution is to choose a good combination of battery capacity, PV-panel area and CHP heat / electricity production ratio. Such a combination is presented in Table 4. In relation to our previous work [22] the CHP heat / electricity production ratio is kept the same, at 2.0, the battery capacity is increased to $4 \mathrm{kWh}$ and the PV-panel area is increased to $22.4 \mathrm{~m}^{2}\left(14 \mathrm{PV}\right.$-panels of $1.6 \mathrm{~m}^{2}, 16 \%$ efficiency). In this case the average daily energy demand from the grid per house in the tested weeks is decreased from $1.5 \mathrm{kWh}$ to $0.3 \mathrm{kWh}$, which is a $80 \%$ reduction. 
Table 4: Equipment configuration and sizes for the neighbourhood.

\begin{tabular}{l|l}
\hline Equipment & Size and configuration \\
\hline CHP unit & $60 \mathrm{~kW}_{\mathrm{th}} / 30 \mathrm{~kW}$ el \\
CHP buffer & $250 \mathrm{kWh}$ \\
Battery & $4 \mathrm{kWh} /$ house \\
PV-panels & $22.4 \mathrm{~m}^{2} /$ house (16\% eff.) \\
\hline
\end{tabular}

Note that, when performing an economic analysis, this method should be extended with a Pareto optimum analysis, specifying the costs (or total costs of ownership) for each option. This will limit the number of possible options to the best trade-offs between minimizing the energy imports and the costs. For reasons stated in the introduction we abstain from such a Pareto optimality analysis in this work.

\section{Results \& Discussion}

In the previous section we have defined the considered neighbourhood and its energy systems and we have determined the proper size of all equipment. In this Section, the behaviour of the neighbourhood microgrid is investigated. Therefore, in this section we evaluate the results of yearly simulations of the microgrid for different coordination mechanisms and some variants of the considered microgrid. Two uncoordinated scenarios, called $N B$ (without batteries) and $N C$ (with batteries) are considered. In these scenarios the different devices have only a local control and do not consider interaction with other devices in their environment. Additionally, two coordinated and optimized cases, both with the batteries included, are simulated. For the control we use profile steering, whereby one scenario uses event-driven profile steering [40] for real-time control (referred to as $P S E$ ), and the other one uses the double-sided auction for real-time control (called $P S A$ ). More details on these two control methods are given in Section 2.4. Furthermore, a variety of the ideal microgrid which uses a realistic battery model instead of an ideal battery model is evaluated. This leads to a variation on the PSE scenario (called PSE-B) in which the ideal battery model is replaced for the realistic battery model [37].

\subsection{Yearly results}

Over the whole year, the average annual electricity demand of a household within the microgrid is $3033 \mathrm{kWh}$, of which $2676 \mathrm{kWh}$ is static load and $357 \mathrm{kWh}$ is consumed by flexible whitegood appliances. The yearly production per household is $8109 \mathrm{kWh}$, of which the PV-panels produce on average $3745 \mathrm{kWh}$ per household and the CHP produces $4364 \mathrm{kWh}$ of electricity per household on an annual basis. The average household heat demand is $8728 \mathrm{kWh}$.

In Figure 8, the load duration curves of the power import/export of the considered microgrid are shown for the four scenarios. As one would expect, the $N B$ case performs worst in terms of autarky, as it has to import both the most energy, but also draws higher peak powers. Adding a battery, with a very simplistic and greedy controller already shows a significant improvement where the peak power of the complete microgrid is reduced 


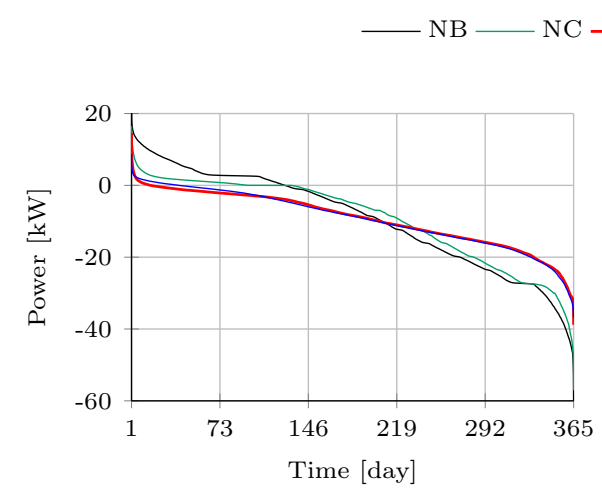

(a) Full load duration curve

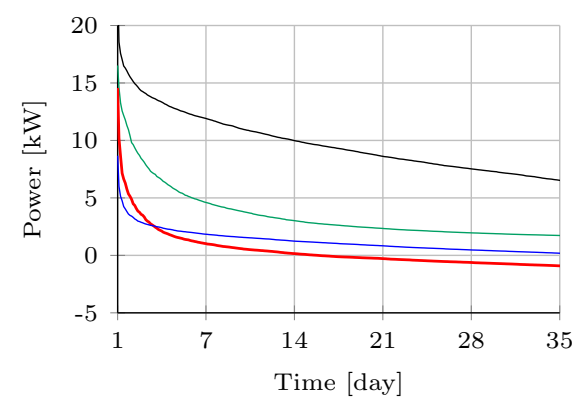

(b) Zoomed load duration curve

Figure 8: Load duration curves of the power demand of the entire microgrid.

from $24.0 \mathrm{~kW}$ to $16.5 \mathrm{~kW}$. Both coordinated cases (PSE and $P S A)$ result in significant improvements, where the more direct method of control $P S A$ allows to reduce the peak power almost completely. On the other hand, PSE is able to use the flexibility better to optimize towards autarky, because of its model predictive nature. With PSE, electricity is imported from the main grid only for $4.3 \%$ of the time, followed by PSA with $10.7 \%$. The $N C$ case has a deficit for significantly more intervals $(31.0 \%)$ and without batteries $(N B)$ the results are even worse $(34.7 \%)$. As expected, without batteries, the DoA (as defined in Section 1) is rather low with $69.7 \%$ for the $N B$ case. When introducing batteries, the DoA significantly increases to $93.2 \%(N C)$. By adding coordination, close to $100 \%$ autarky can be reached, as shown in Table 5 .

Table 5: Statistics of the full year, all values represent the average per household

\begin{tabular}{rccccccc}
\hline & {$[\mathbf{k W h}]$} & {$[\mathbf{k W h}]$} & {$[\%]$} & \multicolumn{4}{c}{ Share in electricity supply [\%] } \\
& Import & Export & DoA & Grid & PV & CHP & BAT \\
\hline NB & 918.2 & 5993.3 & 69.7 & 30.3 & 28.9 & 40.8 & 0.0 \\
NC & 270.8 & 5346.4 & 93.2 & 6.8 & 43.1 & 30.6 & 19.5 \\
PSE & 36.5 & 5111.0 & 99.1 & 0.9 & 41.9 & 42.2 & 15.0 \\
PSA & 66.0 & 5138.4 & 98.4 & 1.6 & 41.6 & 40.9 & 15.9 \\
PSE-B & 61.7 & 5127.4 & 98.4 & 1.6 & 40.6 & 44.2 & 13.6 \\
\hline
\end{tabular}

Table 6: Statistics of the full year, all values represent the average per household

\begin{tabular}{rccccc}
\hline & {$[\%]$} & {$[\mathrm{kWh}]$} & \multicolumn{3}{c}{ Origin of energy [\%] } \\
& Self-consumption & Buffered & Grid & PV & CHP \\
\hline NB & 26.09 & 0.0 & 0.0 & 0.0 & 0.0 \\
NC & 40.88 & 933.6 & 0.0 & 92.1 & 7.9 \\
PSE & 43.66 & 963.9 & 0.0 & 87.7 & 12.3 \\
PSA & 43.39 & 970.8 & 0.0 & 88.3 & 11.6 \\
PSE-B & 42.86 & 874.7 & 0.0 & 86.2 & 13.8 \\
\hline
\end{tabular}


Table 7: Statistics of specific weeks, all values represent the average per household

\begin{tabular}{rccccccc}
\hline & {$[\mathrm{kWh}]$} & {$[\mathrm{kWh}]$} & {$[\%]$} & \multicolumn{3}{c}{ Share in electricity supply [\%] } \\
Week A & Import & Export & DoA & Grid & PV & CHP & BAT \\
\hline NB & 20.48 & 154.43 & 63.92 & 36.08 & 47.88 & 16.04 & 0.00 \\
NC & 3.32 & 137.11 & 95.89 & 4.11 & 59.07 & 11.56 & 25.26 \\
PSE & 0.12 & 135.21 & 99.86 & 0.14 & 64.52 & 16.84 & 18.50 \\
PSA & 0.27 & 133.95 & 99.68 & 0.32 & 63.55 & 14.79 & 21.34 \\
& & & & & & & \\
Week B & Import & Export & DoA & Grid & PV & CHP & BAT \\
\hline NB & 30.77 & 54.17 & 48.21 & 51.79 & 26.93 & 21.28 & 0.00 \\
NC & 11.63 & 35.07 & 85.93 & 14.07 & 45.85 & 14.41 & 25.67 \\
PSE & 2.32 & 26.15 & 97.17 & 2.83 & 48.37 & 24.17 & 24.64 \\
PSA & 8.10 & 30.52 & 90.39 & 9.61 & 46.59 & 20.59 & 23.21 \\
& & & & & & & \\
Week C & Import & Export & DoA & Grid & PV & CHP & BAT \\
\hline NB & 0.26 & 201.80 & 99.57 & 0.43 & 7.95 & 91.62 & 0.00 \\
NC & 0.16 & 201.91 & 99.77 & 0.23 & 17.16 & 78.65 & 3.96 \\
PSE & 0.00 & 203.07 & 100.00 & 0.00 & 5.34 & 91.52 & 3.13 \\
PSA & 0.00 & 202.64 & 100.00 & 0.00 & 5.71 & 90.27 & 4.02 \\
\hline
\end{tabular}

Table 5 also provides information about the origin of the consumed energy. Without batteries, most energy that is locally produced comes from the CHP, which can be explained by the fact that heat demand, and therefore electricity production, is linked strongly to human activity. In the $N C$ case, the batteries are controlled based on the energy balance within the house, which results in a much higher usage of (stored) solar energy. With the coordination system, the share between CHP and PV energy is close to a fifty-fifty division. More notably, by better matching demand and supply, the PSE controlled case is able to achieve the high $99.1 \%$ DoA by using more energy directly from the PV panels and CHP. This results in a lower usage, and thus wearing, of the batteries. To get more insight into the usage of the batteries, in Table 6 we give information on the self consumption of locally produced electricity, on the amount of buffered energy and the origin of the buffered energy. The results show that the batteries are mainly used to store electricity produced by PV, as the electricity production by the CHP can be matched with the demand more easily through the large heat buffer and the simultaneous demand for heat and electricity.

\subsection{Specific weeks}

As seasonal effects play a role in the performance of a microgrid, in this subsection we analyse some of the data also considered in the previous subsection, presented as graphs in Figure 9, the details can be found in Table 7. In the coldest week, week C, the CHP has to run often to fulfil the heat demand (see Figure 9c). In terms of electricity, it thereby produces more than enough energy throughout the day, such that even the uncontrolled case, without batteries, almost reaches a $100 \%$ DoA. In the other two weeks, where the production of $\mathrm{PV}$ becomes more dominant, we see that the DoA significantly drops for $N B$ as it cannot store the electricity generated from $\mathrm{PV}$ to balance the energy mix. As a 
result, during the week with the least energy production, the DoA drops to only $48.2 \%$. Both PSE and PSA are able to maintain a high DoA. However, the full model predictive approach within $P S E$ is clearly better able to keep on matching supply and demand in week B (Figure 9b), still resulting in a $97.2 \%$ DoA during this challenging week. In the coordinated cases, the battery is almost exclusively used to store electricity from PV in both week A and week B.

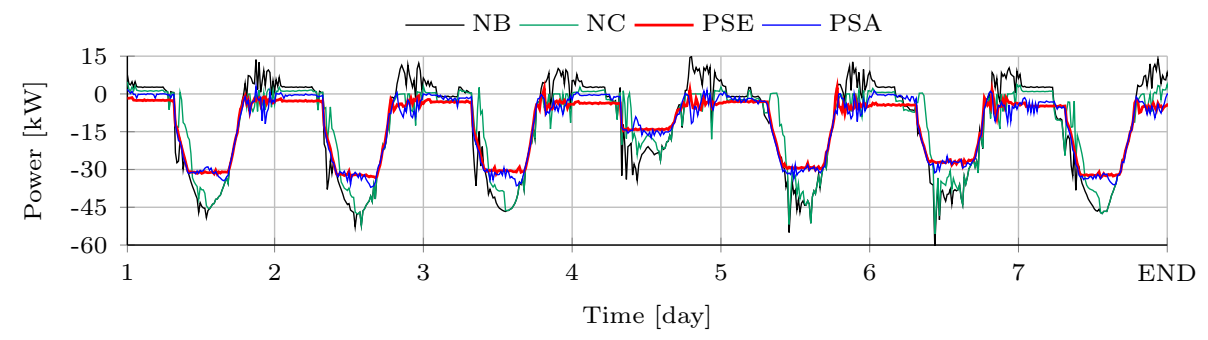

(a) Week A

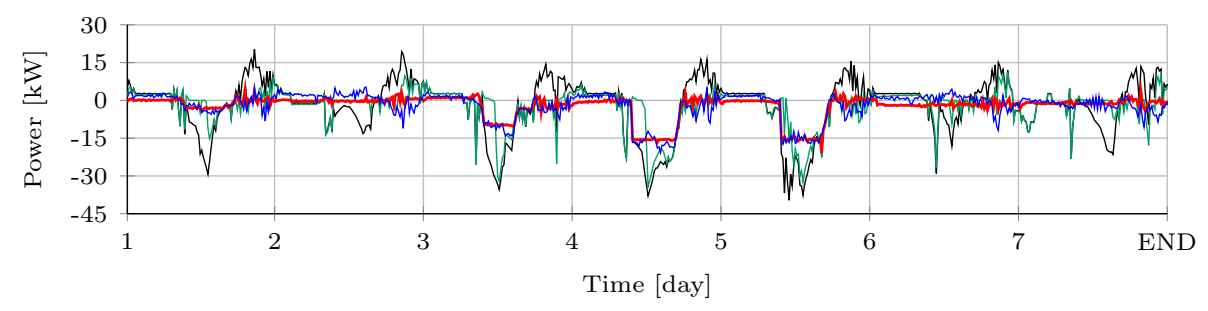

(b) Week B

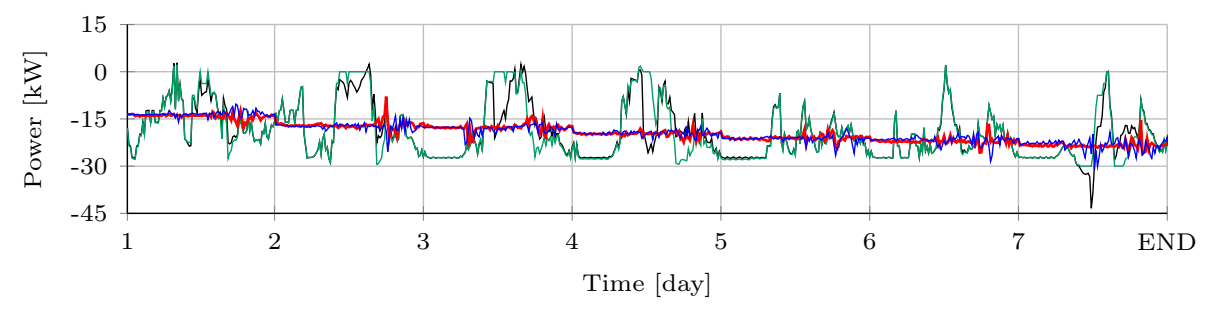

(c) Week C

Figure 9: Simulation results of the total grid power demand for the microgrid in weeks A, B and C. (see Table 3)

\subsection{Planning versus realization}

Profile steering makes use of model predictive control to optimize the energy usage of connected devices. It does so by creating a planning for each device (which then is aggregated for each house) in an online fashion. As such, the accuracy of a planning depends on the accuracy of the predictions and therefore also affects the DoA. Furthermore, online control is applied to resolve prediction and planning errors to ensure that control actions are feasible for the attached devices. The online-control method results in the actual profile, which we refer to as the realization. In this section we compare the 
planning and the realisation to get a better understanding of the differences between the two.

For the PSE case, the annually imported energy per house in the planning was $28.3 \mathrm{kWh}$ with energy import for $4.0 \%$ of the time, whereas in the realization this is $36.5 \mathrm{kWh}$. The results of the simulations show that the planned DoA is slightly better, with $99.3 \%$ compared to the $99.1 \%$ obtained for the realization. For the PSA case, the prediction remarkably overestimates the average energy that each house needs to import, at $94.1 \mathrm{kWh}$ compared to the actual $66.0 \mathrm{kWh}$. Furthermore it is estimated that the house needs to import electricity for $17.8 \%$ of the time. The difference between the planning and the realisation is caused by the fact that the control with the double-sided auction results in different priorities for different devices compared to that of the profile steering based planning. Therefore, flexibility is used in a different way. The planned DoA is also worse with $97.6 \%$ compared to the $98.4 \%$ obtained for the realization.

\subsection{Realistic battery model}

In a real situation the given battery technologies may not be able to provide the relative high power output configured for the ideal battery model. For this, another PSE simulation was done using the realistic DiBu battery model from [37] indicated with PSE-B. Herein, the parameters for a Lead-acid battery obtained in previous work [38] were used and up-scaled to match the $4 \mathrm{kWh}$ battery of the model. As a result, the maximum power rating of $3.7 \mathrm{~kW}$ (both charging and discharging) had to be reduced to a maximum of $1.8 \mathrm{~kW}$ for discharging and a maximum of $0.9 \mathrm{~kW}$ for charging. The results of this simulation are presented in Tables 5, 6 and 7, and shown in Figure 10. Due to the significant lower maximum charging power, the battery is not able to absorb as much solar energy as observed in the ideal PSE case, which results in a drop of the DoA to $98.4 \%$. This decrease is most dominant in the challenging week $B$ where the DoA drops from $99.9 \%$ (ideal battery) to $97.2 \%$ with the realistic battery. But overall, the results show that profile steering usually does not result in high peak discharge and charge powers, such that the realistic battery can closely follow the original planning. The production, consumption and flexibility profiles (Figure 10) also show relative high production and lower consumption power profiles, which is complementary to the (asymmetrical) higher charge power for the realistic battery. Hence, the lower discharge power of the realistic battery model does not lead to problems for the considered household power profiles.

\section{Conclusions}

In this paper the possibilities of operating a neighbourhood (almost) disconnected from the national electricity grid in the Netherlands are explored. For this, we introduced the Degree of Autarky as a measure of how far one can operate a neighbourhood as an islanded microgrid, and hereby expanding upon the concept of soft-islanded microgrids, introduced in [22]. Soft-islanded microgrids are able to fulfil their energy demand most of the time, from locally produced electricity, but remain connected for grid stabilization. Hereby, the level of comfort should be the same as for a normal neighbourhood, so that all heat and electricity demands are met at all times. The neighbourhood we analyse is a futuristic "greenfields" neighbourhood which has features, like e.g. state of the art isolation. Furthermore, each house is equipped with a $4 \mathrm{kWh}$ battery, $22.4 \mathrm{~m}^{2} \mathrm{PV}$ panels 


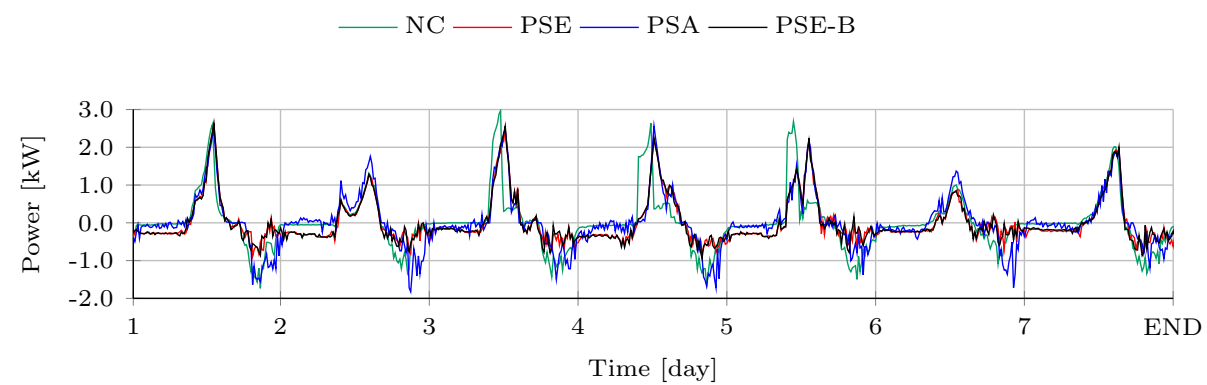

(a) Power

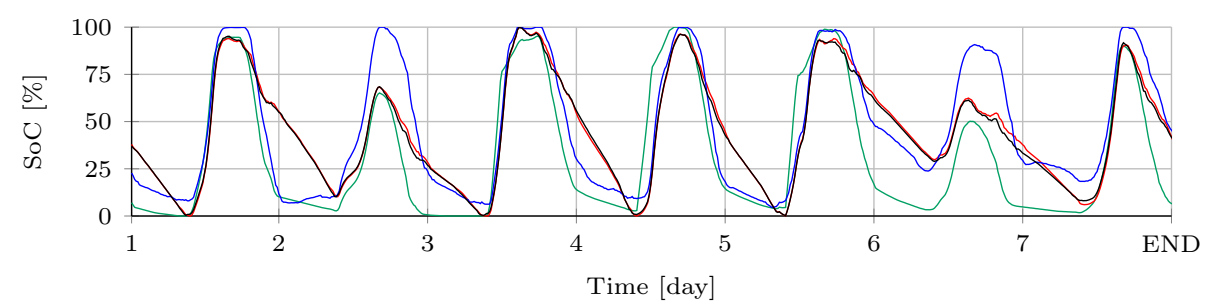

(b) SoC

Figure 10: Battery discharge, charge and state of charge in various simulations for week B. Aggregated data of all batteries.

and the houses share a $60 \mathrm{~kW}_{\mathrm{th}} / 30 \mathrm{~kW}_{\mathrm{el}}$ CHP unit. The sizing of these equipments is detailed in Section 3. As a result of the process of determining the proper sizes of all equipment in the neighbourhood, we conclude that sizing the equipment simultaneously yields better results than determining the proper size of all equipment sequentially due to the interaction between different energy carriers in a MES.

Furthermore, we conclude that the methodology of simultaneous equipment sizing, as demonstrated in Section 3.4, was proven to be quite effective. The selection of "worst case scenarios" (in our case the three weeks defined in Table 3), negates the need for whole year simulations and speeds up the process. The ability to focus on the analysis of only one characteristic value for each simulation (the total weekly grid power demand in $\mathrm{kWh}$ ), results in a quick and comprehensive way to find the best size and configuration of the equipment in the neighbourhood. In this work we focussed on sizing the PVpanel area, the CHP heat to power ratio and the battery capacity, but conceivably more characteristics can be taken into account, e.g. the efficiency of the PV-panels or the size of the CHP buffer. If equipment prices are known, this could also be a powerful tool, e.g. the increase in DoA related to an increase in equipment costs is valuable information for investors and other executives.

The results of the sizing and simulations indicate that either substantial battery storage or overproduction from renewable sources is required to achieve an (nearly) autarkic operating microgrid. The over-all best results are gained in the PSE case, (i.e. batteries are placed in each house and profile steering is applied), with a DoA of 99.1\%. Electricity 
imports from the grid are limited to only $36.5 \mathrm{kWh} /$ year. However, $5.1 \mathrm{MWh} /$ year is still exported to the grid. The results over a whole year indicate that in all cases the PV installations are "over dimensioned" in the sense that less than half of all locally generated energy is consumed locally. Hereby, again the PSE case performs best at $43.7 \%$.

When comparing the PSE-B and PSE scenarios we see that the application of the realistic battery model results in a $1.8 \%$ lower self-consumption, $9.3 \%$ less electricity buffered, a $0.7 \%$ lower DoA and a $40.8 \%$ higher electricity import from the grid. But overall, the observations show that profile steering in general does not result in high discharge peaks and high charge powers, such that the realistic battery can closely follow the original planning. Moreover, the PSE- $B$ yields more realistic results, and answers the question what DoA can be reached for a given battery capacity more accurately.

Generally we conclude that complete islanding of the neighbourhood would be very costly. On the one hand, to reduce the electricity imports an unrealistic amount of electrical energy storage should be installed, most of which will only be used a few hours per year. On the other hand, to reduce the electricity exports, the CHP should produce no more electricity than strictly necessary, which would mean that heat-demands would not be met at all times, which would cause a loss of comfort, which is not acceptable. Therefore, soft-islanding would be a better solution as an average daily import of $0.1 \mathrm{kWh}$ per house can be considered a marginal load for the grid. The huge amounts of overproduction can potentially stress the grid, but for this it is also possible to perform curtailment.

When it comes to the energy mix, the CHP is able to provide electricity when required with a relative small heat buffer as both heat demand and electricity demand usually coincide. This is also indicated by the small share of electricity generated by the CHP stored in the batteries. Therefore, the ratio of heat/electricity generation of the CHP should ideally match the ratio of heat/electricity demand. If such a CHP does not exist, an heat pump can be added to create the appropriate ratio in case of electricity overproduction. For PV, the selection is different as here the battery storage capacity is dominant. However, significant overproduction of electricity by both CHP and PV reduce the required size of the batteries. Concerning the batteries, capacity itself seems to be more important than the power rating, as shown with the realistic DiBu battery model. With only a quarter of the maximum charging power, the microgrid is still able to capture enough electricity produced from PV systems to operate nearly autarkic.

Lastly, we conclude that our methodology of soft-islanding neighbourhoods and simultaneous sizing of the necessary equipment leads to interesting and useful insights on the technical feasibility of different configurations of a neighbourhood MES. These technical results could be used as input for economic feasibility studies and evaluations. To successfully perform such evaluations it is important to know the state-of-the-art of the technology and the actual (trends in) investment and operational costs. A few examples for reviews of the state-of-the-art are the work by Zubi et al. [42] for ( $L i-i o n)$ batteries, the works by Martinez et al. [43] and Murugan et al. [44] for CHPs and the work by Candelise et al. in [45] for PV-panels. The economic feasibility is also heavily influenced by local regulations, subsidies and feed-in tarriffs as surveyed in [46, 47]. Note that, as a result of fast-changing costs (investment, installation, maintenance, ...) laws, pricing 
schemes and subsidy regulations, the results of such an economic evaluation would be highly valuable for the specific location and time-frame under consideration, but far less valuable for other regions and time-frames.

\section{Future work}

The research has also resulted in various topics for future work to improve the optimization of soft-islanded microgrids. Foremost is the difference between short-term and long-term optimization. Within this research, we have only executed short-term planning by optimizing for the next two days using a receding horizon. However, with seasonal storage possibilities, including long-term objectives may result in a better performance during challenging days with little generation. The export of electricity could reduced in future work by investigating the addition of electric vehicles that charge using the excess electricity produced by the CHP. Alternatively power to gas equipment could be installed which uses the excess electricity to create hydrogen for further seasonal storage, which can be stored and used again locally to fuel the CHP or fuel-cell electric vehicles.

Control in this paper was executed in intervals of 15 minutes, which is too slow for a microgrid. Online control approaches, such as the robust approach presented in [48], may be integrated to react to fast fluctuations of e.g. intermittent PV production. This will not only smoothen out short peaks, it will also result in possibilities to slightly increase the DoA by better matching the demand with these fluctuations. Challenges in the field of economics, sociology and lawmaking remain open with the pure technological oriented study presented. For example, future research should be conducted on a fair and accepted reward mechanism where participants are rewarded appropriately for helping the local community.

The determination of the optimal size of the equipment in the neighbourhood, as discussed in Section 3.4, was carried out by manually changing and evaluating the effects of different parameters for the equipment. This process could be automated by including a local search over the parameter space using the simulation carried out by DEMKit itself as a function evaluation. If proper boundary conditions are applied this should yield the optimal size for all equipment. This process could be expanded to include the various costs (investment, installation, etc) and benefits of the equipment to yield a financially optimal equipment size. Note that such a method significantly increases the number of simulations needed and therefore requires a lot of computational power for execution. Furthermore, to accurately size the components, the control algorithms must be extended to reflect local markets and regulations. Implementing such algorithms, as well as an automated sizing algorithms is part of future work.

\section{Acknowledgements}

The authors would like to thank the Dutch national program TKI Switch2Smargrids (project Smart Grid Evolution), and the Dutch national program TKI iDeego (project ORTEP) and RVO for their support. The authors would also like to acknowledge the anonymous reviewers for their constructive comments on this work. 


\section{References}

[1] Ministerie van Economische Zaken, Energieagenda - Naar een $\mathrm{CO}_{2}$ arme energievoorziening, 2016.

[2] ECN, Energie-Nederland, Netbeheer Nederland, Energietrends, 2016.

[3] Netbeheer Nederland, Net voor de toekomst - Een vooruitblik op de energievoorziening in 2050, 2017

[4] Netbeheer Nederland, Grids for the future - A look ahead towards the energy supply in $2050,2017$.

[5] P. Siano, Demand response and smart grids - A survey, Renewable and Sustainable Energy Reviews 30 (2014) 461-478.

[6] K. McKenna, A. Keane, Discrete elastic residential load response under variable pricing schemes, in: IEEE PES Innovative Smart Grid Technologies, Europe, 2014, pp. 1-6.

[7] S. Nykamp, M. G. C. Bosman, A. Molderink, J. L. Hurink, G. J. M. Smit, Value of storage in distribution grids: competition or cooperation of stakeholders?, IEEE Transactions on Smart Grid 4 (2013) 1361-1370.

[8] R. H. Lasseter, Smart Distribution: Coupled Microgrids, Proceedings of the IEEE 99 (2011) 1074-1082.

[9] B. Lasseter, Microgrids [distributed power generation], in: 2001 IEEE Power Engineering Society Winter Meeting. Conference Proceedings (Cat. No.01CH37194), volume 1, 2001, pp. 146-149.

[10] D. E. Olivares, A. Mehrizi-Sani, A. H. Etemadi, C. A. Cañizares, R. Iravani, M. Kazerani, A. H. Hajimiragha, O. Gomis-Bellmunt, M. Saeedifard, R. Palma-Behnke, G. A. Jiménez-Estévez, N. D. Hatziargyriou, Trends in microgrid control, IEEE Transactions on Smart Grid 5 (2014) 1905-1919.

[11] J. M. Guerrero, M. Chandorkar, T. L. Lee, P. C. Loh, Advanced control architectures for intelligent microgrids: part i: decentralized and hierarchical control, IEEE Transactions on Industrial Electronics 60 (2013) 1254-1262.

[12] A. L. Dimeas, N. D. Hatziargyriou, Operation of a multiagent system for microgrid control, IEEE Transactions on Power Systems 20 (2005) 1447-1455.

[13] A. Molderink, V. Bakker, M. G. C. Bosman, J. L. Hurink, G. J. M. Smit, Management and Control of Domestic Smart Grid Technology, IEEE Transactions on Smart Grid 1 (2010) 109-119.

[14] M. E. T. Gerards, H. A. Toersche, G. Hoogsteen, T. van der Klauw, J. L. Hurink, G. J. M. Smit, Demand side management using profile steering, in: PowerTech, 2015 IEEE Eindhoven, 2015, pp. $1-6$.

[15] P. Mancarella, MES (multi-energy systems): An overview of concepts and evaluation models, Energy 65 (2014) 1-17.

[16] N. Good, E. Martinez Cesena, C. Heltorp, P. Mancarella, A transactive energy modelling and assessment framework for demand response business cases in smart distributed multi-energy systems, Energy (2018)

[17] E. A. M. Cesena, P. Mancarella, Energy systems integration in smart districts: robust optimisation of multi-energy flows in integrated electricity, heat and gas networks, IEEE Transactions on Smart Grid (2018) 1-1.

[18] S. Long, O. Marjanovic, A. Parisio, Demand smoothing in multi-energy systems using model predictive control, in: 2018 IEEE PES Innovative Smart Grid Technologies Conference Europe (ISGT-Europe), 2018, pp. 1-6.

[19] K. X. Perez, M. Baldea, T. F. Edgar, Integrated smart appliance scheduling and HVAC control for peak residential load management, in: 2016 American Control Conference (ACC), 2016, pp. 1458-1463.

[20] Vereniging Aardehuis, [Online] Available: http://aardehuis.nl/nl/aardehuizen/visie-missie, 2019. Last accessed on 31-7-2019.

[21] C. Statline, Energiebalans; aanbod en verbruik, sector, [Online] Available: https://opendata.cbs.nl, 2017. Last accessed on 18-6-2019.

[22] K. X. Perez, M. Baldea, T. F. Edgar, G. Hoogsteen, R. P. van Leeuwen, T. van der Klauw, B. Homan, J. Fink, G. J. M. Smit, Soft-islanding a group of houses through scheduling of CHP PV and storage, in: 2016 IEEE International Energy Conference (ENERGYCON), Leuven, 2016, pp. 1-6.

[23] J. Salom, A. J. Marszal, J. Widén, J. Candanedo, K. B. Lindberg, Analysis of load match and grid interaction indicators in net zero energy buildings with simulated and monitored data, Applied Energy 136 (2014) 119-131.

[24] I. Sartori, A. Napolitano, K. Voss, Net zero energy buildings: A consistent definition framework, Energy and Buildings 48 (2012) 220-232.

[25] E. Palacios-Garcia, A. Moreno-Muñoz, I. Santiago, I. Moreno-Garcia, M. Milanés-Montero, PV 
hosting capacity analysis and enhancement using high resolution stochastic modeling, Energies 10 (2017) $1-22$.

[26] V. Bakker, Triana: a control strategy for Smart Grids: Forecasting, planning \& real-time control, Ph.D. thesis, University of Twente, 2012.

[27] A. Molderink, On the three-step control methodology for Smart Grids, Ph.D. thesis, University of Twente, 2011.

[28] G. Hoogsteen, A Cyber-Physical Systems Perspective on Decentralized Energy Management, Ph.D. thesis, University of Twente, 2017.

[29] K. Kok, The PowerMatcher: smart coordination for the smart electricity grid, Ph.D. thesis, Vrije Universiteit Amsterdam, 2013.

[30] J. Torriti, A review of time use models of residential electricity demand, Renewable and Sustainable Energy Reviews 37 (2014) 265-272.

[31] U. Wilke, F. Haldi, J.-L. Scartezzini, D. Robinson, A bottom-up stochastic model to predict building occupants time-dependent activities, Building and Environment 60 (2013) 254-264.

[32] M. A. López-Rodríguez, I. Santiago, D. Trillo-Montero, J. Torriti, A. Moreno-Munoz, Analysis and modeling of active occupancy of the residential sector in Spain: An indicator of residential electricity consumption, Energy Policy 62 (2013) 742-751.

[33] J. Widén, M. Lundh, I. Vassileva, E. Dahlquist, K. Ellegård, E. Wäckelgård, Constructing load profiles for household electricity and hot water from time-use-data-modelling approach and validation, Energy and Buildings 41 (2009) 753-768.

[34] J. Widén, E. Wäckelgård, A high-resolution stochastic model of domestic activity patterns and electricity demand, Applied Energy 87 (2010) 1880-1892.

[35] G. Hoogsteen, A. Molderink, J. L. Hurink, G. J. M. Smit, Generation of flexible domestic load profiles to evaluate Demand Side Management approaches, in: 2016 IEEE International Energy Conference (ENERGYCON), Leuven, 2016, pp. 1-6.

[36] KNMI, Uurgegevens van het weer in Nederland, [Online] Available: http://www.knmi.nl, 2014 Last accessed on 27-10-2017.

[37] B. Homan, M. V. ten Kortenaar, J. L. Hurink, G. J. M. Smit, A realistic model for battery state of charge prediction in energy management simulation tools, Energy 171 (2019) 205-217.

[38] B. Homan, V. M. J. J. Reijnders, G. Hoogsteen, J. L. Hurink, G. J. M. Smit, Implementation and Verification of a Realistic Battery Model in the DEMKit Simulation Software, in: 2018 IEEE PES Innovative Smart Grid Technologies Europe, Sarajevo, 2018, pp. 1-6.

[39] R. van Leeuwen, Towards $100 \%$ renewable energy supply for urban areas and the role of smart control, Ph.D. thesis, University of Twente, 2017.

[40] G. Hoogsteen, A. Molderink, J. L. Hurink, G. J. M. Smit, Asynchronous event driven distributed energy management using profile steering, in: 2017 IEEE Manchester PowerTech, 2017, pp. 1-6.

[41] T. van der Klauw, Decentralized Energy Management with Profile Steering - Resource Allocation Problems in Energy Management, Ph.D. thesis, University of Twente, PO Box 217, 7500 AE Enschede, The Netherlands, 2017. doi:10.3990/1.9789036543019.

[42] G. Zubi, R. Dufo-López, M. Carvalho, G. Pasaoglu, The lithium-ion battery: State of the art and future perspectives, Renewable and Sustainable Energy Reviews 89 (2018) 292-308.

[43] S. Martinez, G. Michaux, P. Salagnac, J.-L. Bouvier, Micro-combined heat and power systems (micro-CHP) based on renewable energy sources, Energy Conversion and Management 154 (2017) $262-285$.

[44] S. Murugan, B. Horák, A review of micro combined heat and power systems for residential applications, Renewable and Sustainable Energy Reviews 64 (2016) 144-162.

[45] C. Candelise, M. Winskel, R. J. Gross, The dynamics of solar pv costs and prices as a challenge for technology forecasting, Renewable and Sustainable Energy Reviews 26 (2013) 96 - 107.

[46] L. Dusonchet, E. Telaretti, Comparative economic analysis of support policies for solar pv in the most representative eu countries, Renewable and Sustainable Energy Reviews 42 (2015) 986 - 998

[47] B. K. Sahu, A study on global solar pv energy developments and policies with special focus on the top ten solar pv power producing countries, Renewable and Sustainable Energy Reviews 43 (2015) $621-634$.

[48] M. E. T. Gerards, J. L. Hurink, Robust peak-shaving for a neighborhood with electric vehicles, Energies 9 (2016). 